\title{
Left ventricular outflow tract obstruction coexisting with ventricular septal defect
}

\author{
D F SHORE, J SMALLHORN, J STARK, C LINCOLN, M R DE LEVAL
}

\author{
From the Brompton Hospital and The Hospital for Sick Children, London
}

SUMmARY Between January 1973 and March 1982, 13 patients have been identified who had in addition to a ventricular septal defect, a discrete narrowing of the left ventricular outflow tract. Two patients had tetralogy of Fallot and one a complete atrioventricular canal defect. Two patients had previously undergone repair of a juxtaductal coarctation and one patient banding of the pulmonary artery. Eleven patients, 10 of whom had left ventricular outflow tract obstruction (mean left ventricular aortic pressure gradient $=71 \cdot 2 \pm 35 \cdot 3 \mathrm{mmHg}$ ) underwent excision of a fibromuscular shelf. A myotomy was performed in addition in six patients. The left ventricular aortic pressure gradient measured postoperatively was less than $15 \mathrm{mmHg}$ in all but one patient. In seven patients the shelf was excised at the time of closure of the ventricular septal defect, but in four patients the left ventricular outflow tract obstruction became manifest later. Two further patients not operated upon have a discrete narrowing of the left ventricular outflow tract with a left ventricular aortic pressure gradient of 5 and $30 \mathrm{mmHg}$. Two dimensional echocardiographic assessment of the left ventricular outflow tract was performed in eight patients and compared with left ventricular angiography. Echocardiography clearly visualised the fibromuscular shelf and its relation to the ventricular septal defect in each case. In three of the eight patients the shelf was not seen on the left ventricular angiogram.

Assessment of the left ventricular outflow tract in patients with ventricular septal defect by echocardiography will allow recognition of left ventricular outflow tract abnormalities. When a discrete fibromuscular shelf is identified we recommend its excision at the time of closure of the ventricular septal defect. This should be performed even in the absence of a left ventricular aortic pressure gradient to prevent the progressive development of left ventricular outflow tract obstruction and associated myocardial changes.

Ventricular septal defect coexisting with left ventricular outflow tract obstruction is a rare anomaly. Lauer et al. ${ }^{1}$ found 10 cases among a pathological collection of 722 hearts with congenital cardiac anomalies. In recent reports the incidence of this combined anomaly varied between 5 and $20 \%$ of all patients with discrete subaortic obstruction. ${ }^{2-6}$ Not surprisingly, the incidence was highest in a series of paediatric patients with discrete subaortic obstruction reported by Newfeld et al. in 1976. ${ }^{4}$ These authors also drew attention to the fact that the presence of a subaortic stenosis was frequently masked by the symptoms and signs of the ventricular septal defect. Failure to recognise the presence of left ventricular outflow tract obstruction at the time of a ventricular septal defect can result in postoperative morbidity and mortality.

The purpose of this report is to review the case Accepted for publication 29 June 1982 histories, the haemodynamic, angiographic, and echocardiographic data of those patients in whom this combined lesion was found to exist, with particular emphasis on the difficulties and importance of accurate preoperative diagnosis.

\section{Subjects and methods}

Between January 1973 and March 1982, 13 patients were seen at the Brompton Hospital and The Hospital for Sick Children, London, who in addition to a ventricular septal defect had a discrete narrowing of the left ventricular outflow tract diagnosed either before or after closure of a ventricular septal defect.

Group 1 consisted of four patients (whose mean age at the time of excision of the fibromuscular shelf was 12 years 2 months) in whom the diagnosis of subaortic obstruction (mean left ventricular aortic pressure gradient $97 \mathrm{mmHg}$ ) was made after closure of the 
ventricular septal defect. The second operation was performed for its relief at a mean of 5 years 9 months after closure of the ventricular septal defect.

Group 2 consisted of seven patients (whose mean age at the time of excision of the fibromuscular shelf was 5 years 8 months) in whom the diagnosis of subaortic obstruction (mean left ventricular aortic pressure gradient $46 \mathrm{mmHg}$ ) was made before closure of a ventricular septal defect.

Group 3 consisted of two patients who have a discrete narrowing of the left ventricular outflow tract but as yet have not undergone surgery. One of these has no gradient across the left ventricular outflow tract and the other has a gradient of $30 \mathrm{mmHg}$. Their ages are 14 months and $2 \frac{1}{2}$ years, respectively.

One patient in group 1 previously underwent a pulmonary artery banding and one patient in group 2 had previously undergone a repair of a juxtaductal coarctation.

Four patients in group 1 , and seven in group 2, have undergone excision of a discrete fibromuscular shelf. Three in each of groups 1 and 2 had a myotomy performed in addition to excision of the fibromuscular shelf.

Two patients in groups 1 and 2 had aortic regurgitation. In the patient in group 1 , this was the result of a small perforation, probably a congenital malformation, in the non-coronary cusp which was repaired. The second case had had bacterial endocarditis with more extensive perforation of the non-coronary cusp, which necessitated valve replacement. Two further patients had minor abnormalities of the aortic valve without functional effect.

The ventricular septal defect was described as perimembranous inlet in one patient, perimembranous infundibular in six patients, perimembranous trabecular in one patient, and muscular infundibular in two patients. The ventricular septal defect was described as perimembranous in two patients without further elaboration. Two patients with perimembranous infundibular defects had tetralogy of Fallot. One patient had a complete atrioventricular canal defect.

The morphological features of the subaortic shelf were similar to those described in other series. The shelf took the form of either a crescent or a complete ring. Like others, we found that the distance between the suboartic shelf and the aortic valve ring varied, but in addition the ventricular septal defect could lie proximally or distally to it. The position of the fibromuscular shelf and its relation to the ventricular septal defect or patch was determined from either the description of the operative findings or from the crosssectional echocardiogram. The shelf was above the defect in four patients and below the defect in seven patients. In the remaining two patients the position of the membrane in relation to the ventricular septal defect is unknown.

All patients underwent cardiac catheterisation before closure of the ventricular septal defect. The four patients in group 1 had a right heart catheterisation only before the ventricular septal defect was closed, but all patients in groups 2 and 3 had both a right and left heart catheterisation. All the patients in group 1 had a left heart catheterisation before excision of the subaortic obstruction. Serial cardiac catheterisation was performed in seven patients: four patients in group 1 , two patients in group 2, and one patient in group 3 .

Cross-sectional echocardiographic assessment of the left ventricular outflow tract was performed in three patients in group 1 , three patients in group 2 , and both patients in group 3 . Contemporary left ventricular angiograms and cross-sectional echocardiograms are available for comparison in eight patients. In five patients these were performed before closure of the ventricular septal defect and in three patients after closure of the ventricular septal defect but before excision of the fibromuscular shelf.

\section{Results}

HOSPITAL MORTALITY

There were no hospital deaths in this series either after closure of the ventricular septal defect, or relief of subaortic obstruction alone, or when the two procedures were performed together.

REDUCTION OF LEFT VENTRICULAR AORTIC GRADIENT

A satisfactory reduction in the left ventricular aortic pressure gradient to less than $15 \mathrm{mmHg}$ was recorded intraoperatively after resection of the fibromuscular shelf in all but one patient. In this patient a rise in the left ventricular-aortic pressure gradient was recorded. This patient who was aged 5 years 4 months at the time of operation had in addition to a fibromuscular shelf a more diffuse fibromuscular narrowing of the left ventricular outflow tract. As much of the fibrous tissue and endocardium of the left ventricular outflow tract was excised as was practicable without risking damage to the conduction tissue or the mitral valve.

The mean length of follow-up in the 10 patients whc have undergone excision of the fibromuscular shelf is three years. Nine of these patients are alive and wel and have no evidence of recurrent left ventricular outflow tract obstruction. The patient referred ts above who had a high residual gradient underwent : Konno procedure five and a half years after the initia operation. One patient who had a postoperativ gradient of $10 \mathrm{mmHg}$ has developed recurrent sever tunnel subaortic obstruction with a left ventricula aortic pressure gradient of $80 \mathrm{mmHg} 18$ months afte the excision of the subaortic shelf and is awaitin 


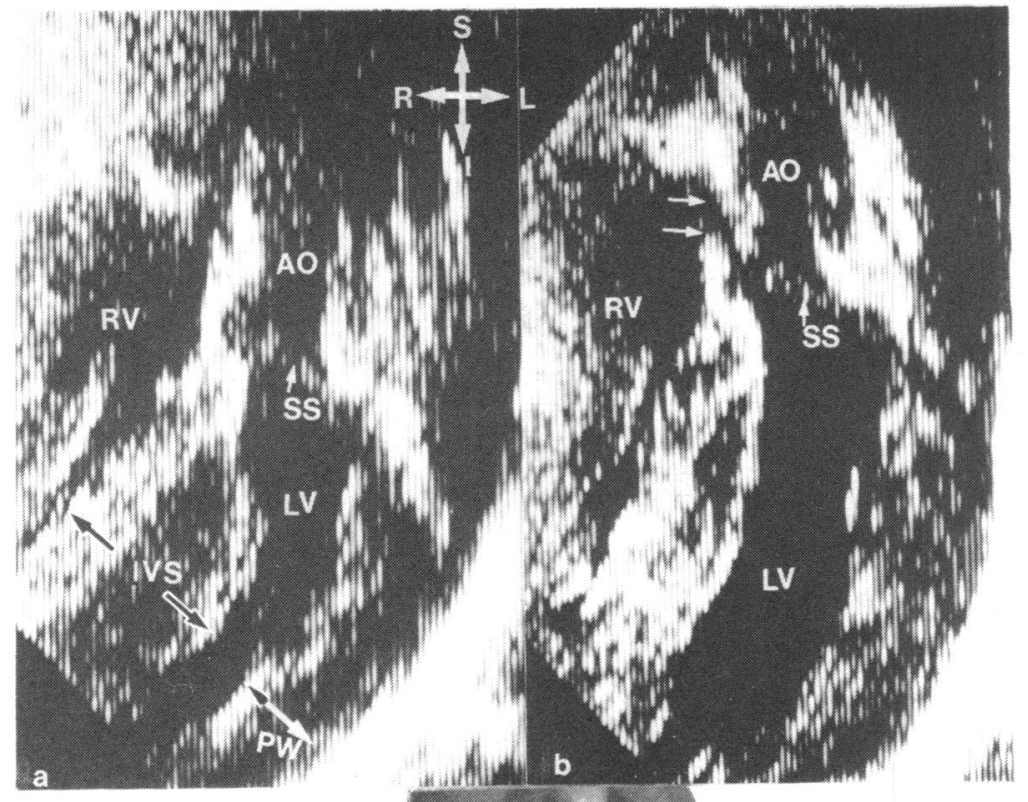

Fig. 1. (a) This picture shows the subaortic shelf in a case with ventricular septal defect associated with hypertrophic obstructive cardiomyopathy. Note the thickness of the septum in relation to the posterior left ventricular wall. (b) This image is from the same case, to show the relation of the subaortic shelf to the ventricular septal defect. Note the shelf lies above the defect. The white arrows indicate the ventricular septal defect. (c) The angiocardiogram from the same patient also shows the subaortic shelf which lies above the ventricular septal defect. AO, aorta; PW, posterior left ventricular wall; IVS, interventricular septum; LV, left ventricle; $R V$, right ventricle; $S S$, subaortic shelf.

further surgery (Fig. 1).

Review of the echocardiographic data disclosed that in the eight patients in whom this was performed it clearly showed the morphology of the left ventricular outflow tract, the position of the obstructing tissue, and the relation of this tissue to the ventricular septal defect in each case (Fig. 1, 2, and 3). In three of the five cases in which cross-sectional echocardiography was performed before closure of the ventricular septal defect the abnormality of the left ventricular outflow tract could be clearly seen on the echocardiogram but not on the left ventricular angiogram (Fig. 3 and 4). These patients had gradients of 0,0 , and $30 \mathrm{mmHg}$, and therefore in two a left ventricular aortic withdrawal would not have indicated any abnormality of the left ventricular outflow tract.

\section{Discussion}

Ventricular septal defect in association with subaortic stenosis is a rare combination of congenital cardiac anomalies. In a pathological collection of 722 hearts with congenital cardiac anomalies, Lauer et al. ${ }^{1}$ found 10 cases with this combination of defects. They categorised these cases according to the size of the ventricular septal defect and its relation to the level of obstruction. This enabled them to make reliable predictions about differences in pressures in the aorta, pulmonary arteries, and ventricular chambers when this information was not available from catheterisation. The subaortic obstruction was the result of a variety of causes.

In only one heart, however, was the anatomical 


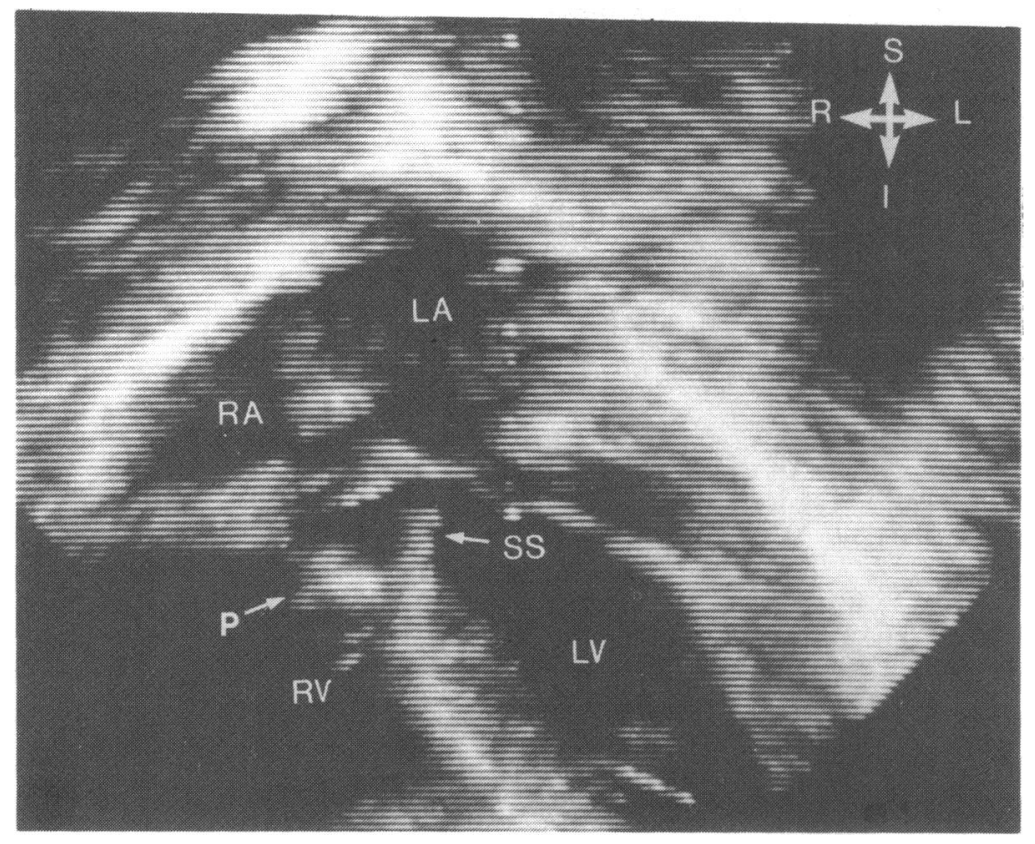

Fig. 2. This is an apical four chamber cut in a case with subaortic obstruction detected after patch closure of a large inlet ventricular septal defect. Note the dense echoes from the patch and the large subaortic shelf. The left ventricular free wall and interventricular septum are also hypertrophied. LA, left atrium; RA, right atrium; $P$, patch. For the rest of the abbreviations see Fig 1 .
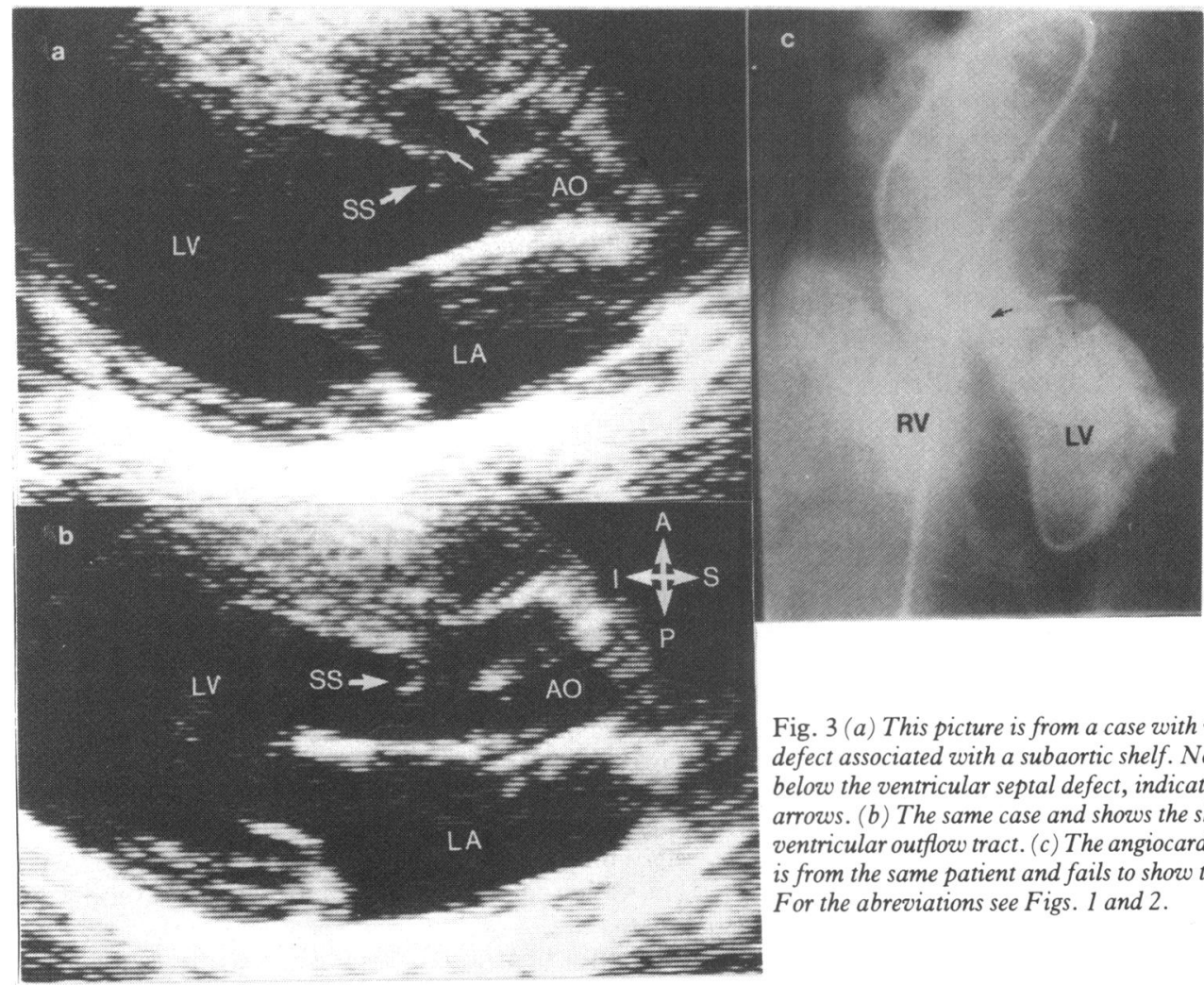

Fig. 3 (a) This picture is from a case with ventricular septal defect associated with a subaortic shelf. Note the shelf lies below the ventricular septal defect, indicated by the two white arrows. (b) The same case and shows the shelf in the left ventricular outflow tract. (c) The angiocardiogram on the right is from the same patient and fails to show the subaortic shelf. For the abreviations see Figs. 1 and 2. 


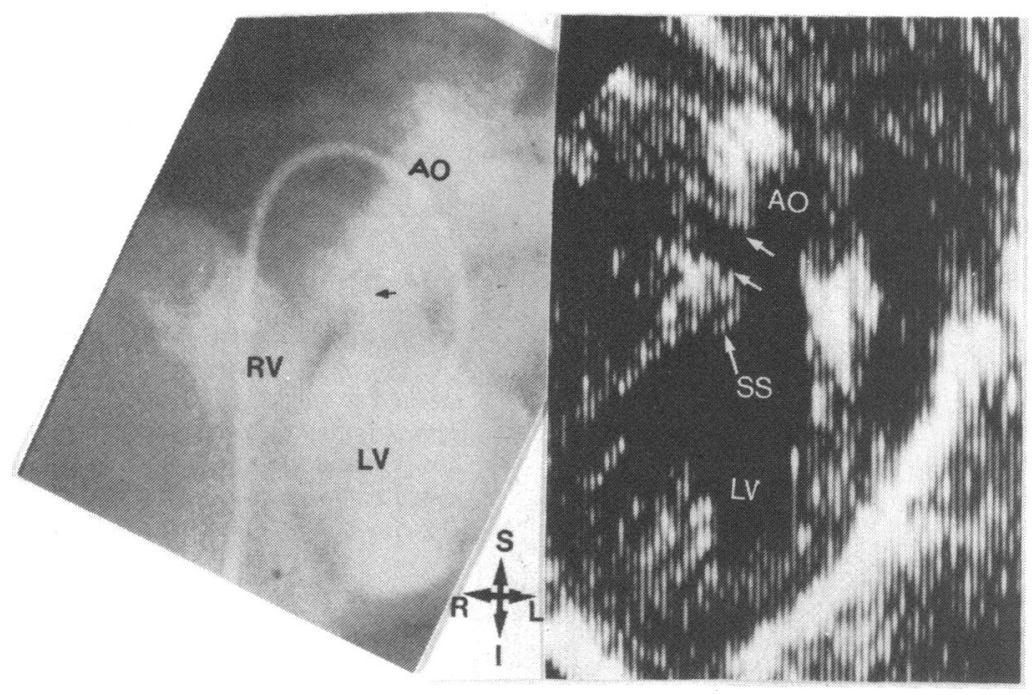

Fig. 4. This is from a case with ventricular septal defect and subaortic shelf, where there was no detectable gradient at cardiac catheterisation. Note the shelf lies below the defect, indicated by the arrows. The angiocardiogram in the same case fails to show the shelf. For the abbreviations see Fig. 1 .

obstruction primarily caused by a fibromuscular shelf or ring. The other causes of obstruction included abnormal insertion of the tension apparatus of the atrioventricular valve in a heart with a complete atrioventricular septal defect, origin of the aorta and pulmonary artery from the right ventricle with a restrictive ventricular septal defect with and without pulmonary stenosis, hypertrophy of the anterolateral muscle bundle of Moulaert and OppenheimerDekker, ${ }^{7}$ and posterior displacement of the infundibular septum. The last abnormality is part of a complex of cardiac congenital anomalies, which includes ventricular septal defect and biventricular origin of the pulmonary trunk, and is associated with anomalies of the aortic arch. This complex had previously been described by Becu et al. in 1955.8

In this report we have confined discussion to those cases of subaortic stenosis resulting primarily from a discrete fibromuscular shelf or ring appearing in the setting of atrioventricular and ventriculoarterial concordance. We have not included in this paper cases of ventricular septal defect and subaortic obstruction arising from the other causes listed above because in those the obstruction is primarily the result of a congenital morphological abnormality of the left ventricular outflow tract. We believe these cases have a different natural history and the obstruction is not amenable to direct surgical relief. A possible exception would be those cases where the obstruction is caused by hypertrophy of the muscle bundle of Moulaert, but we have not encountered a case where this anomaly occurred with ventricular septal defect. Though left ventricular outflow tract obstruction resulting from a discrete fibromuscular shelf may occur in transposition of the great arteries, surgical considerations differ because the obstruction is subpulmonary.

Several reports have pointed to the progressive nature of the obstruction primarily caused by a discrete fibromuscular shelf. In a number of cases a complete surgical cure can be effected by its excision. The natural history of subaortic obstruction resulting from a fibromuscular shelf when associated with a ventricular septal defect is probably no different from those cases where it occurs in isolation. From a clinical standpoint the importance of this association lies in the fact that the subaortic stenosis may be masked by the ventricular septal defect. Failure to recognise the presence of the subaortic obstruction, however, may lead to postoperative mortality and morbidity after closure of the ventricular septal defect or the need for a further operation for its relief. On the other hand, patients with a combined lesion may present earlier than those with isolated subaortic obstruction because of the symptoms and signs of the ventricular septal defect. This affords the surgeon the opportunity of early surgical intervention to prevent progression of the subaortic obstruction if it is recognised. Newfeld et al. ${ }^{4}$ and Somerville et al. ${ }^{5}$ have pointed to the advantages of earlier recognition and surgical treatment in order to minimise the development of myocardial degeneration and a superimposed dynamic element to the left ventricular outflow tract obstruction. In our series, eight of the nine patients who had a significant gradient preoperatively had a satisfactory reduction after excision of the fibromuscular shelf. In the tenth patient there was no gradient between the left ventricle and aorta, and the shelf was excised as a prophylactic measure. The one patient who had a high residual 


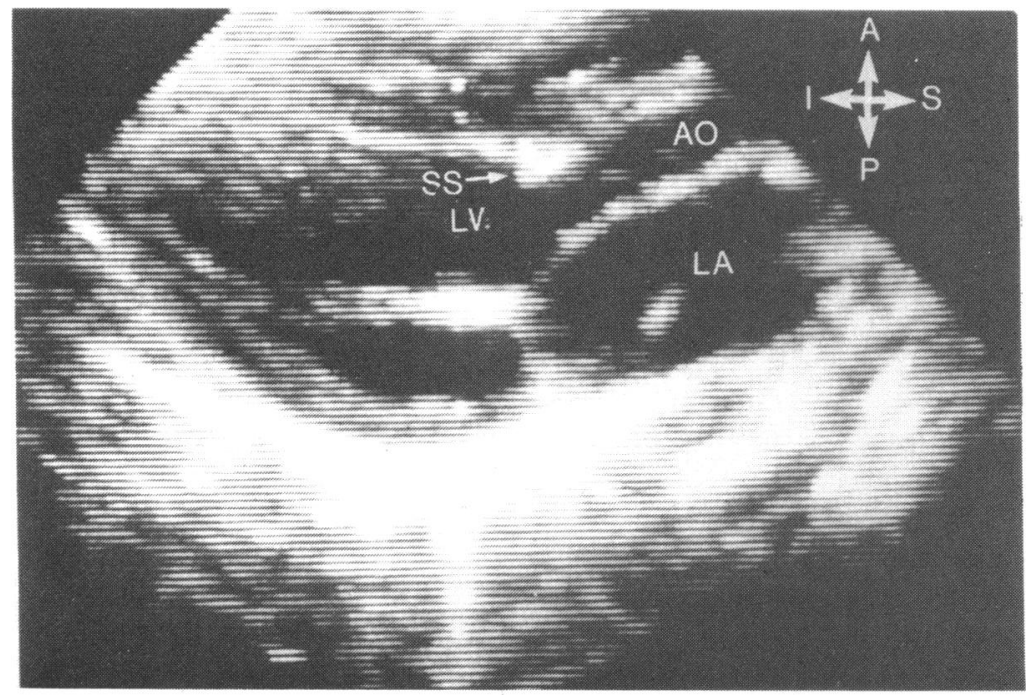

Fig. 5. This is a parasternal long axis cut in a case with a ventricular septal defect and associated subaortic ridge. Note the protrusion into the left ventricular outflow tract is smooth, unlike in Fig. 4. This patient had a low gradient across the outflow tract at cardiac catheterisation. The posterior free wall and interventricular septum are not hypertrophied. For the abbreviations see Fig. 1.

gradient had, in addition to a fibromuscular shelf, a more diffuse fibrous change of the left ventricular outflow tract. He required a Konno procedure five years later after the progressive development of the left ventricular outflow tract obstruction. One other patient had severe recurrent left ventricular outflow tract obstruction. Before operation she had the angiographic and echocardiographic features of hypertrophic obstructive cardiomyopathy (Fig. 1), but had no gradient measured intraoperatively after excision of the fibromuscular shelf. She would serve to illustrate two important points that Somerville et al. ${ }^{5}$ made in a recent report on the fate of patients with fixed subaortic obstruction after surgical removal. They found that intraoperative pressure measurements taken after excision of the fibromuscular shelf were no guide to the likelihood of redeveloping fixed subaortic obstruction or the persistence of dynamic obstruction probably because of the depressed state of the myocardium after bypass. They also found that the most reliable prognostic sign that dynamic obstruction would persist was the morphology of the left ventricle on angiography; those patients with excessive septal hypertrophy in systole or an eccentric muscle lump always had postoperative dynamic obstruction. Dynamic obstruction was most common in those operated upon in the second decade of life when the majority of patients in their series presented.

Of the eight patients, however, who had a low postoperative gradient between the left ventricle and the aorta, seven have no evidence of recurrent fixed or persistent dynamic obstruction though the follow-up in the majority of patients is short.

Redevelopment of fixed subaortic obstruction and the ability to reform a fibromuscular ring or shelf, histopathologically similar to the one excised, led Somerville et al. to speculate on the aetiology. They described two hearts examined by Becu taken from children aged 9 and 15 months who died of lung infections associated with a large shunt at ductal level. In both these hearts there was a minute non-obstructive fleshy ridge beneath the aortic valve cusp. In profile this ridge bears close morphological resemblance to the structure seen projecting from the lower edge of the ventricular septal defect seen on the cross-sectional echocardiogram (but not on the angiogram) in a $2 \frac{1}{2}$ year old patient who has a gradient of $30 \mathrm{mmHg}$ across the left ventricular outflow tract (Fig. 5). Similar but slightly more filamentous lesions have been identified on the cross-sectional echocardiogram in two further patients who at catheterisation had no measured gradient across the left ventricular outflow tract and had no angiographically identifiable abnormality (Fig. 3 and 4). In one of these patients a fibrous shelf was excised from the left ventricular side of the lower margin of the ventricular septal defect during its transatrial closure.

Early excision of such material may prevent the progressive development of left ventricular outflow tract obstruction. In the presence of this abnormal tissue the increased flow in patients with left to right shunt may increase the turbulence and accelerate the development of obstruction, thus making early recognition even more important. Newfeld et al. ${ }^{4}$ have proposed, and we agree, that if such abnormal fibromuscular tissue projecting into the outflow tract can be identified in patients with ventricular septal defects it should be excised, even in the absence of a measured gradient, in order to prevent the later development of subaortic obstruction. 
The difficulty lies in its identification. It appears from our initial experience that cross-sectional echocardiography is a superior method of identifying such an abnormality of the left ventricular outflow tract in patients with ventricular septal defect when compared with angiography. In three of the seven patients who had both a left ventricular angiogram and a cross-sectional echocardiogram the abnormality of the left ventricular outflow tract was visible only on the echocardiogram, and in two of these patients there was no left ventricular aortic pressure gradient measured.

In patients with isolated left ventricular outflow tract obstruction difficulty in clearly defining the lesion angiographically has previously been reported.9-13 Lesions directly beneath the aortic valve may be interpreted as valvular obstruction, and angiography does not always allow differentiation between obstruction caused by a fibromuscular ridge, a diffuse tunnel obstruction, or idiopathic hypertrophic obstructive cardiomyopathy. Wilcox et al..$^{13}$ showed the superiority of cross-sectional echocardiography in the recognition and characterisation of discrete subaortic obstruction. In patients with an associated ventricular septal defect difficulties of angiographic recognition are compounded by contrast medium crossing the defect. This problem does not occur with cross-sectional echocardiography. By combining the cuts it is possible accurately to describe the position of the ventricular septal defect ${ }^{14}$ and its relation to the obstruction. Newfeld et al. ${ }^{4}$ pointed out that there might be difficulties of angiographic definition and that the pressure measurements might be equally misleading. In particular, if the obstruction is below a ventricular septal defect through which a catheter is passed from the right ventricle to the aorta, absence of a pressure gradient between right ventricle and aorta may be erroneously presumed to rule out subaortic obstruction. If the obstruction is above the defect, the latter will decompress the left ventricle, and, if flow through the aortic valve is for any reason reduced, lead to underestimation of the severity of the obstruction. There is also the possibility that closure of the defect will exacerbate the subaortic stenosis. In patients with a concordant ventriculoarterial connection, however, it is unlikely that closure of the ventricular septal defect in itself can ever produce subaortic obstruction except perhaps in those rare cases where a hypertrophied muscle bundle of Moulaert lies directly opposite a large ventricular septal defect or where the membrane extends on to the anterior mitral leaflet. In these cases there is an unrestrictive communication between the ventricular chambers on the one hand and between the right ventricle and the aorta on the other. In 1960 Lauer $e t$ al. ${ }^{1}$ described how insertion of the ventricular septal patch in itself produced subaortic obstruction.

In summary we recommend that particularly careful cross-sectional echocardiographic assessment of the left ventricular outflow tract is performed in all cases of ventricular septal defect before proceeding to surgical repair. Routine simultaneous measurement of left ventricular and aortic pressures after closure of a ventricular septal defect is equally vital. In addition we feel that any fibromuscular shelf that is identified should be excised even in the absence of a measured gradient between the left ventricle and the aorta.

\section{References}

1 Lauer RM, Dushane JW, Edwards JE. Obstruction of left ventricular outlet in association with ventricular septal defect. Circulation 1960; 22: 110-25.

2 Shariatzadeh AN, King H, Girod D, Shumacker HB Jr. Discrete subaortic stenosis. A report of 20 cases. $\mathcal{F}$ Thorac Cardiovasc Surg 1972; 63: 258-62.

3 Champsaur G, Trusler GA, Mustard WT. Congenital discrete subvalvular aortic stenosis. Surgical experience and long term follow-up in 20 paediatric patients. Br Heart f 1973; 35: 443-6.

4 Newfeld EA, Muster AJ, Paul MH, Idriss FS, Riker WL. Discrete subvalvular aortic stenosis in childhood. Study of 51 patients. Am $\mathcal{F}$ Cardiol 1976, 38: 53-61.

5 Somerville J, Stone S, Rosa D. Fate of patients with fixed subaortic stenosis after surgical removal. Br Heart $\mathcal{F} 1980$; 43: $629-47$.

6 Katz NM, Buckley MJ, Liberthson RR. Discrete membranous subaortic stenosis. Report of 31 patients, review of the literature and delineation of management. Circulation 1977, 56: 1034-8.

7 Moulaert AJ, Oppenheimer-Dekker A. Anterolateral muscle bundle of the left ventricle, bulboventricular flange and subaortic stenosis. Am $\mathcal{F}$ Cardiol 1976; 37: 78-81.

8 Becu LM, Newlon Tauxe W, Dushane JW, Edwards JE. A complex of congenital cardiac anomalies: ventricular septal defect, biventricular origin of the pulmonary trunk, and subaortic stenosis. Am Heart f 1955; 50: 901-11.

9 Lundquist CB, Amplatz K. The subvalvular aortic jet. Radiology 1965; 85: 635-44.

10 Slezák P, Steinhart L, Procházka J, Endrys J, Jurin I. The angiographic appearances of subvalvular aortic stenosis. Br F Radiol 1965; 38: 350-5.

11 Freimanis AK, Wooley CF, Meckstroth CV, Molnar W. Roentgenographic aspects of congenital left ventricular outflow tract obstruction. $A \mathcal{F R}$ 1965; 95: 573-91.

12 Deutsch V, Shem-Tov A, Yahini JH, Neufeld HN. Subaortic stenosis (discrete form): classification and angiocardiographic features. Radiology 1971; 101: 275-86.

13 Wilcox WD, Seward JB, Hagler DJ, Mair DD, Tajik AJ. Discrete subaortic stenosis. Two dimensional echocardiographic features with angiographic and surgical correlation. Mayo Clin Proc 1980; 55: 425-33.

14 Sutherland GR, Godman MJ, Smallhorn JF, Guiterras P, Anderson RH, Hunter S. Ventricular septal defects. Two dimensional echocardiographic and morphological correlations. Br Heart f 1982; 47: 316-28.

Requests for reprints to Dr Marc de Leval, Thoracic Unit, The Hospital for Sick Children, Great Ormond Street, London WC1N 3JH. 